\title{
Interleukin-8 holds promise to serve as a molecular adjuvant in DNA vaccination model against Streptococcus iniae infection in fish
}

\author{
Erlong Wang ${ }^{1, *}$, Bo Long ${ }^{1, *}$, Kaiyu Wang ${ }^{1,2}$, Jun Wang ${ }^{1}$, Yang $\mathrm{He}^{1}$, Xingli Wang ${ }^{1}$, \\ Qian Yang ${ }^{1}$, Tao Liu' ${ }^{1}$, Defang Chen ${ }^{3}$, Yi Geng ${ }^{1,2}$, Xiaoli Huang ${ }^{3}$, Ping Ouyang ${ }^{1}$ and \\ Weimin Lai ${ }^{1}$ \\ ${ }^{1}$ Department of Basic Veterinary, College of Veterinary Medicine, Sichuan Agricultural University, Chengdu, Sichuan, China \\ ${ }^{2}$ Key Laboratory of Animal Disease and Human Health of Sichuan Province, Sichuan Agricultural University, Chengdu, \\ Sichuan, China \\ ${ }^{3}$ Department of Aquaculture, College of Animal Science and Technology, Sichuan Agricultural University, Chengdu, Sichuan, \\ China \\ * These authors have contributed equally to this work
}

Correspondence to: Kaiyu Wang, email: kywang1955@126.com

Keywords: aquaculture, bacterial diseases, DNA vaccine, interleukin-8, molecular adjuvant, Immunology and Microbiology Section, Immune response, Immunity

Received: August 20,2016 Accepted: November 15, $2016 \quad$ Published: November 30, 2016

\section{ABSTRACT}

DNA vaccines had been widely used in animal models against various viral infections, while it was not so convincing for many infectious diseases especially bacterial disease in aquaculture. Interleukin-8(IL-8) as one of the CXC chemokines, its immunological role and adjuvant potential which had been proved in mammals were rarely reported in fish species. In this study, recombination plasmid pcDNA3.1/IL8(pcIL-8) was conducted and the capacity of IL-8 as molecular adjuvant was explored from several aspects by co-injecting with a DNA vaccine encoding a-enolase(pcENO) against Streptococcus iniae infection in channel catfish. The results suggested that co-injection of pcIL-8 with DNA vaccine increased the innate immunity and specific antibody levels, as well as increased the immune-related genes involving in proinflammatory response, humoral and cellular immunity. Moreover, pcIL-8 enhanced the immunoprotection of pcENO with the relative percent survival(RPS) of $60 \%$ to $80 \%$ against $S$.iniae infection at 4 week post vaccination(p.v.), with the significantly higher RPS of $73.33 \%$ in pcENO+pcIL-8 group compared with that of pcENO alone $(53.33 \%)$ at challenge test of 8 weeks p.v. Taken together, these results indicate pcIL-8 as a molecular adjuvant co-injected with DNA vaccine not only improves the immunoprotection but also maintains long period of immunity for channel catfish against S.iniae infection. Our study signifies that IL-8 holds promise to serve as a potential adjuvant in DNA vaccines against bacterial infections for long time.

\section{INTRODUCTION}

Aquaculture as one of the most vibrant sector of the global food system has shown a rapid growth in the last decades and with such growth comes the need for effective control measures against increasingly diseases. Traditionally, controlling the disease and infection in aquaculture relies mainly on the use of antibiotics and antimicrobial compounds. However, several problems including antibiotic resistance and antibiotic residues have arisen due to the indiscriminate use of antibiotics which are not acceptable and even not permitted in some countries [1]. Therefore, seeking for the safe and efficacious vaccines is critical for a sustainable development of the aquaculture industry.

Compared with traditional inactivated and attenuated live vaccines, DNA vaccines consisting of naked plasmid DNA that will result in gene expression of immunoprotective antigen in vivo against fish diseases particularly viral pathogens, have made major advances over the last decade and become one of the most promising vaccine preparations in aquaculture [1]. Despite DNA 
vaccines used in fish have proven particularly efficacious against some viral diseases [2-7], only one DNA vaccine for aquaculture fish has been licensed for sale [8], and it is not so convincing for a number of other viral infections and bacterial disease. Scientists are now focused on improving and increasing the potency of DNA vaccines, which include the improvements of vectors and the covaccination of molecular adjuvants such as cytokines with the antigen to enhance the immune responses $[9,10]$.

Cytokines as a family of low molecular weight and secretory proteins are closely associated with the proinflammatory responses against invasive pathogens and the key regulators of the host immune system [11]. Several studies reported that the use of cytokines as vaccine adjuvants to augment the initial host responsiveness has been widely explored in mammals, and many cytokine genes like IL-1 $\beta$ and TNF- $\alpha$ have been identified in many fish species and many details concerning their immunological role are also demonstrated [11]. Chemokines, or chemotactic cytokines, are a family of cytokines involved in lymphocyte trafficking and immune surveillance within the immune system and served to attract leucocytes to particular sites [12]. Interleukin-8 (IL8 ) as one of the first CXC chemokines to be discovered in fish has been cloned and characterized in few fish species [13-17], while the immunological role of IL-8 has not been achieved and details concerning its adjuvant potential are still lacking in these species.

Channel catfish (Ictalurus punctatus) as the most extensively cultured food-fish species has suffered from widespread disease outbreaks in recent years due to various bacterial pathogens such as Streptococcus iniae, a Gram-positive fish pathogenic bacterium with a broad host range [18-20]. Besides, channel catfish has been served as a classical model for the study of comparative immunology and research on the characterization and immunological role of its cytokines should help elucidate the immune system network in fish [17]. In this study, we further explore the adjuvant potential of IL- 8 of channel catfish from several aspects by co-injecting with a DNA vaccine encoding $\alpha$-enolase (pcENO) against Streptococcus iniae infection based on our previous report that IL-8 hold promise for use as potential immunopotentiator against bacterial infections but insufficient to extend the immune protection for long time in a subunit vaccine model [21].

\section{RESULTS}

\section{Cloning, sequencing and eukaryotic expression plasmid of IL-8}

The channel catfish full length IL-8 gene was successfully PCR-amplified with $360 \mathrm{bp}$ in length (Figure 1A), which contained a complete open reading frame (ORF) and a Kozak sequence at the N-terminus and $6 \times$ histidine tags sequence at the $\mathrm{C}$-terminus. The IL-8 gene encode a 117 amino acid (a.a.) sequence with a predicted signal peptide (1 24 a.a.) and a conserved Chemokine_CXC domain belonging to Chemokine superfamily (30 88 a.a.). Moreover, three consensus motifs named as ELR-like motif which the leucine residue
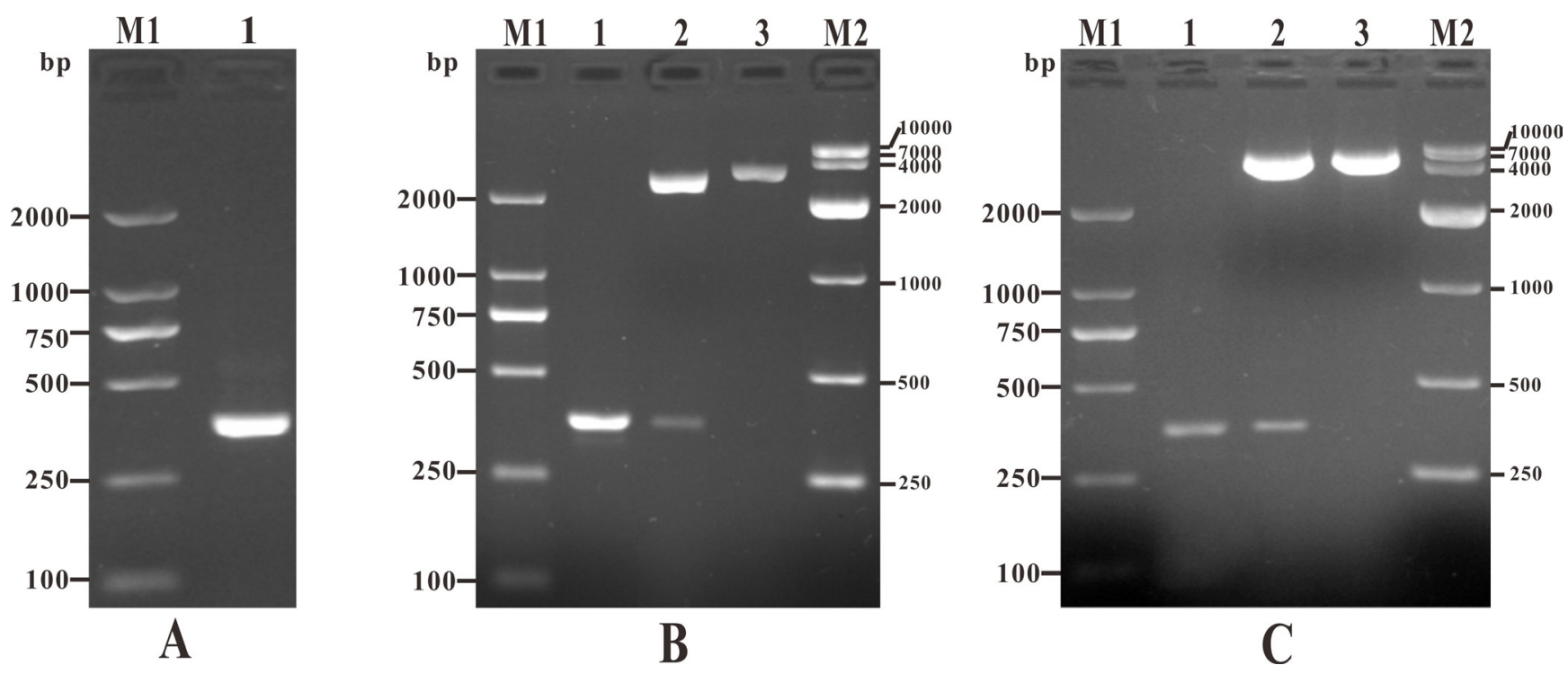

Figure 1: PCR amplification of IL-8 gene, detection of recombinant plasmid T-IL-8 and pcIL-8. A. PCR amplification of IL-8 gene. M1: DNA marker (DL2000); lane 1: PCR product of IL-8 gene (360bp). B. Identification of recombinant plasmid T-IL-8 with PCR and enzyme digestion. M1: DNA marker (DL2000); lane 1: PCR identification of T-IL-8; lane 2: digestion identification of T-IL-8 with Hind III and EcoR I; lane 3: digestion identification with EcoR I; M2: DNA marker (DL10000). C. Identification of recombinant plasmid pcIL-8 with PCR and enzyme digestion. M1: DNA marker (DL2000); lane 1: PCR identification of pcIL-8; lane 2: digestion identification of pcIL-8 with Hind III and EcoR I; lane 3: digestion identification with EcoR I; M2: DNA marker (DL10000). 
was not present, $\mathrm{CXC}$ motif and GPH motif were also found in the Chemokine_CXC domain of channel catfish IL-8 locating at the N-terminal of 30 31, 32 34 and $56 \sim 58$ a.a. respectively (Figure 2 ). In addition, to construct the eukaryotic expression plasmid, IL-8 was successfully cloned into pMD19-T (Figure 1B) and inserted in vector pcDNA3.1 (Figure 1C).
PCR detection of plasmid DNA in vaccinated fish

To confirm the presence of the pcIL- 8 plasmid in muscle tissues at different time intervals, PCR was performed with specific primers. A PCR product in length of $360 \mathrm{bp}$ was detected from DNA extracted from muscle tissues at 7, 14, 28 and 56 days p.v. in pcIL-8 group and pcENO+pcIL-8 group. No amplification was detected in DNA extracted from tissues of the PBS, pcDNA3.1 and pcENO group (Figure 3).

\begin{tabular}{lllllllllllllllllll}
$M$ & $E$ & $A$ & $A$ & $T$ & $L$ & $T$ & $V$ & $L$ & $P$ & $L$ & I & I & F & A & $L$ & $T$ & $A$ \\
\hline
\end{tabular}

61 ATACTTTGTGAAGgATGGGGAGAAGGAAAAGCAGAGCGTTGTTTCTGCCAAAAGAAAGCA

121 GTGGAAAAGGTAAGACCTGCACTTGTGAAGAAGTTTGAGGTATTCCCTCCAAGTGCCTCC

41 V

181 TGTTCAAATACTGAGATCATATTATCTCTGAAGCAAGGAATGAAGGTCTGCCTGGATCCA

$\begin{array}{llllllllllllllllllllll}61 & C & S & N & T & E & \text { I } & \text { I } & L & S & L & K & Q & G & M & K & V & C & L & D & P\end{array}$

241 GAAGGAAACCAAGGACAGAAATCTTAACTGGACAGAAACTAAAGATAAATTCCAAGGGA

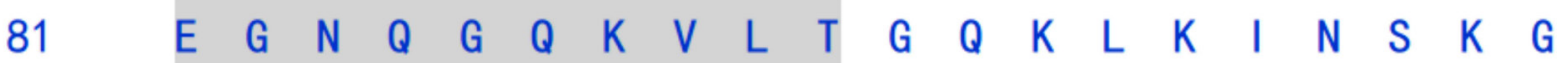

\section{AGAAGGGGAAAGAAGCAGAAGAAGATCAAACAGCAAAACCACCACCACCACCACCACTGA}

$\begin{array}{llllllllllllllllllllllll}101 & \mathrm{R} & \mathrm{R} & \mathrm{G} & \mathrm{K} & \mathrm{K} & \mathrm{Q} & \mathrm{K} & \mathrm{K} & \mathrm{I} & \mathrm{K} & \mathrm{Q} & \mathrm{Q} & \mathrm{N} & \underline{\underline{\mathrm{H}}} & \mathrm{H} & \mathrm{H} & \mathrm{H} & \mathrm{H} & \mathrm{H}\end{array} *$

Figure 2: Nucleotide sequence and derived amino acid sequence of channel catfish IL-8. In the nucleotide sequence, underline presented a Kozak consensus sequence, double underline presented a $6 \times$ histidine tags sequence, the shadow presented initiation codon (ATG) and termination codon (TGA). In the amino acid sequence, underline indicated the predicted signal peptides, shadow regions indicated the conserved Chemokine_CXC domain, the ELR-like motif, CXC motif and GPH motif were indicated by black, blue and red box, respectively, double underline indicated the $6 \times$ histidine tags.

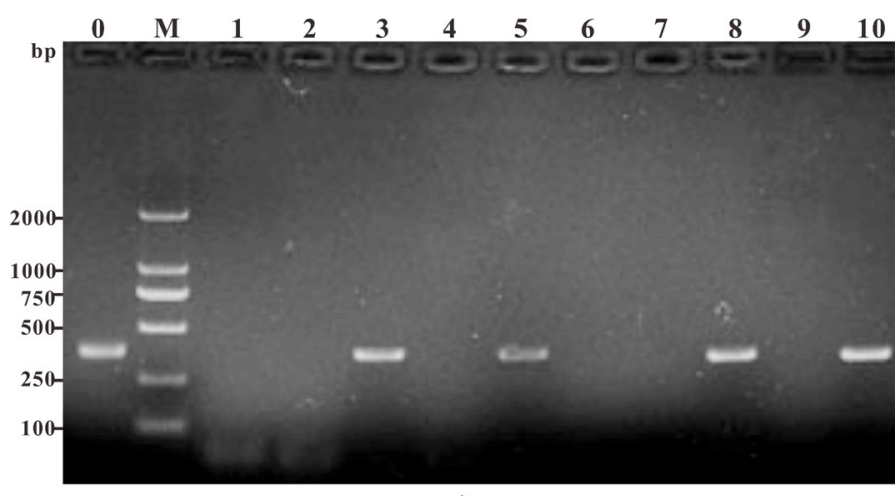

A

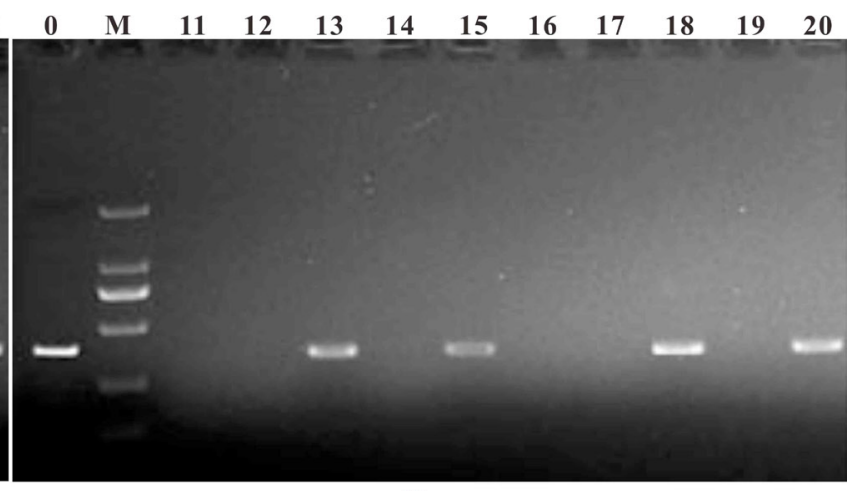

B

Figure 3: PCR detection of plasmid DNA in muscle of the vaccinated fish. Channel fish were treated with PBS (lane 1, 6, 11, 16), or vaccinated with pcDNA3.1 (lane 2, 7, 12, 17), pcIL-8 (lane 3, 8, 13, 18), pcENO (lane 4, 9, 14, 19) and pcENO+pcIL-8 (lanes 5, $10,15,20)$ respectively. Muscle samples were taken and used for DNA extraction from the vaccinated fish at 7 days (lanes 1-5), 14 days (lanes 6-10), 28 days (lanes 11-15) and 56 days (lanes 16-20) p.v. respectively. Lane 0 (positive control): PCR product of plasmid pcIL-8, lane M: DNA markers (DL2000). 


\section{Transcription analysis and expression of DNA vaccines}

Transcription analysis of ENO gene and exogenous IL-8 gene in muscle tissues of the vaccinated fish was performed by RT-PCR at 14 days p.v. The transcription of ENO gene was detected in the injected muscle of pcENO and pcENO+pcIL-8 group, but not in PBS, pcDNA3.1 and pcIL-8 group. Simultaneously, the transcription of exogenous IL- 8 gene was detected in the injected muscle of pcIL- 8 and pcENO+pcIL- 8 group, not in PBS, pcDNA3.1 and pcENO group (Figure 4). Similarly, to examine whether exogenous IL-8 was expressed at translation level in the vaccinated fish, immunohistochemistry analysis was performed, which showed that the production of exogenous IL-8 was detected in muscle tissue of fish vaccinated with plasmid pcIL-8 groups (pcIL-8 and pcENO+pcIL-8 group) at 14 , 28 and 56 days p.v., but not in that of fish vaccinated with no plasmid pcIL-8 groups (PBS, pcDNA3.1 and pcENO group) (Figure 5).

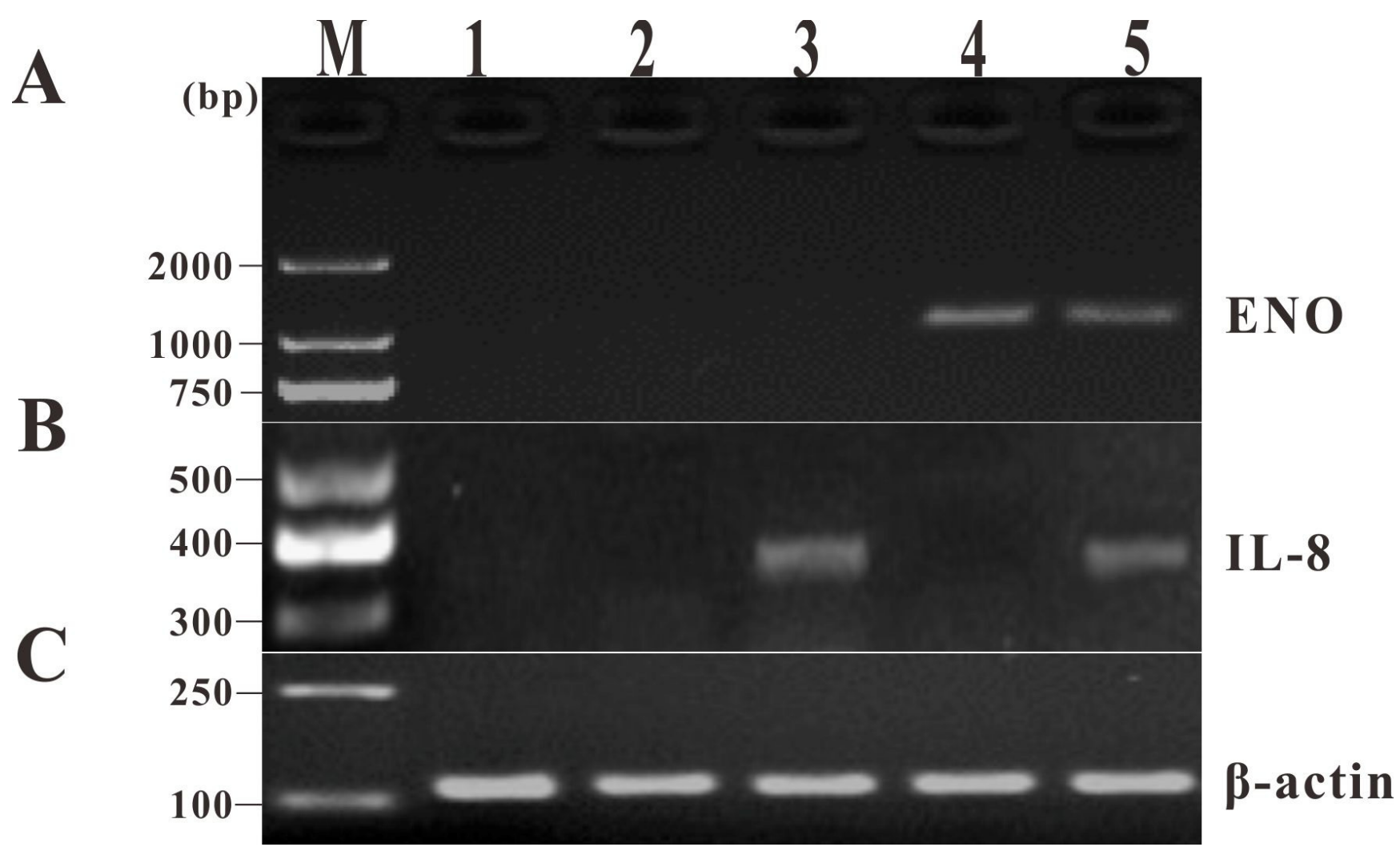

Figure 4: RT-PCR analysis of transcription of ENO gene and exogenous IL-8 gene in muscle tissues of the vaccinated fish at 14 days p.v. A. ENO gene. B. Exogenous IL-8 gene. C. $\beta$-actin gene. M: DNA marker; lane $1 \sim 5$, muscle injected with PBS, pcDNA3.1, pcIL-8, pcENO and pcENO+pcIL-8, respectively. 
pcENO+pcIL-8 was notably higher than that of pcENO except 1 day p.v., with the highest ACH50 activity occurring at 14 days p.v (Figure 6B).

\section{Specific serum antibody response}

The specific antibody in serum of vaccinated fish were assessed by ELISA at different days p.v. No specific anti-rENO antibody was detected in PBS, pcDNA3.1 and pcIL-8 groups during all the experimental time (Figure 7). Compared with above three groups, specific antibody was observed significantly $(p<0.05)$ in pcENO and pcENO+pcIL-8 groups from 7 to 56 days p.v. Moreover, pcENO+pcIL-8 stimulated the production of antibody significantly higher $(p<0.05)$ than pcENO at 7, 14, 21 , 28,42 and 56 days p.v. respectively, with the highest antibody level both peaking at 28 days p.v. (Figure 7).

\section{Expression of immune-related genes}

qRT-PCR was performed to investigate the effect of vaccination on the expression of immune-related genes encoding interleukin $1 \beta$ (IL-1 $\beta$ ), tumor necrosis factor- $\alpha$ (TNF- $\alpha$ ), CXC Chemokine Ligand 10 (CXCL10), major histocompatibility complex class I $\alpha$ (MHC I $\alpha$ ) and II $\beta$ (MHC II $\beta)$, CD4-L2, CD8 $\alpha$ and interferon- $\gamma$ (IFN- $\gamma$ ) with $\beta$-actin (ACTB) as an internal control. The results showed that in fish of group pcIL-8, pcENO and

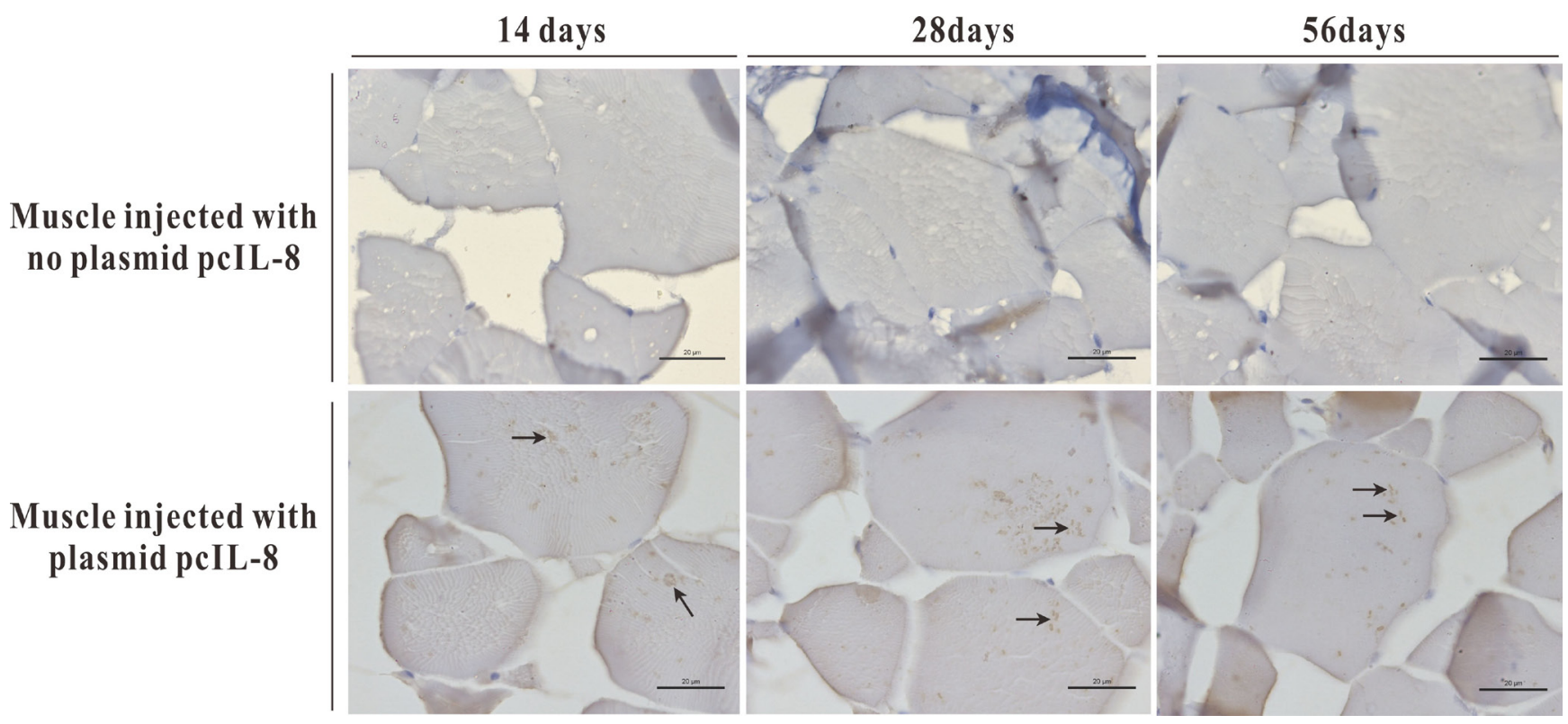

Figure 5: Immunohistochemistry analysis of exogenous IL-8 expressed in muscle tissues of the vaccinated fish at 14, 28 and 56 days p.v. Bar $=20 \mu \mathrm{m}$.
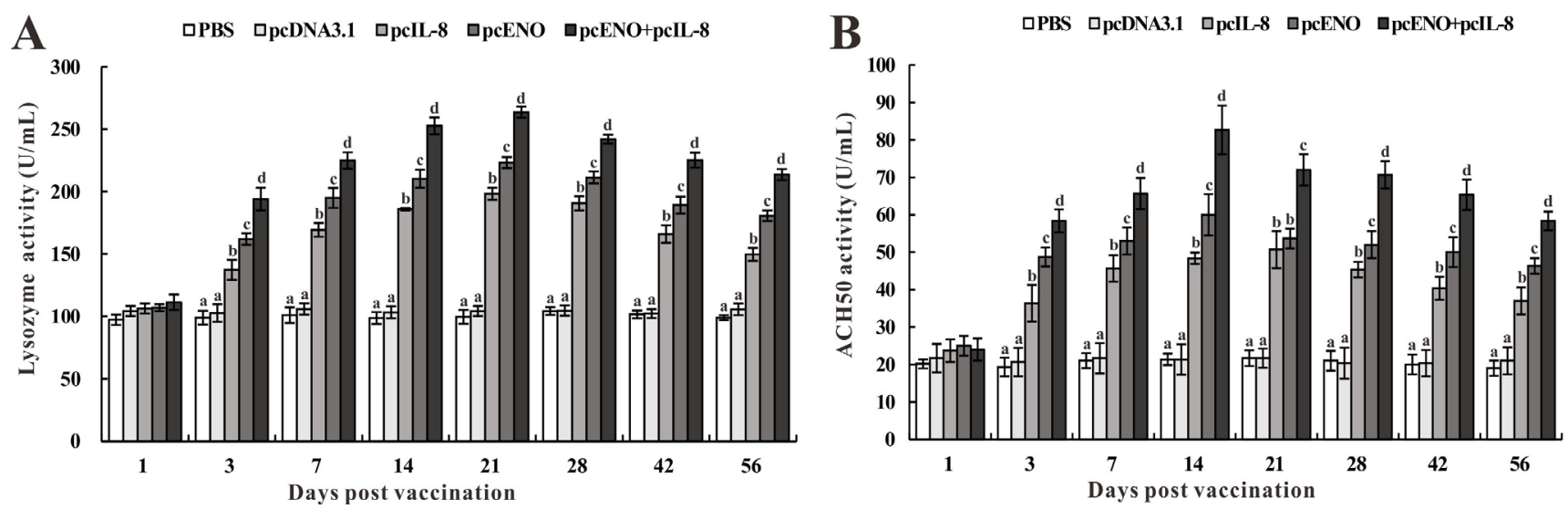

Figure 6: Serum lysozyme activity A. and ACH50 activity B. of vaccinated fish. Sera were collected at different time points p.v. from the fish vaccinated with PBS (control), pcDNA3.1, pcIL-8, pcENO and pcENO+pcIL-8. Data are presented as means \pm SD $(n=5)$. Differences analysis between groups were determined using one-way ANOVA and Duncan's test. Different letters mean that the data of different groups in the same time differ significantly $(p<0.05)$. The same as below. 
pcENO + pcIL- 8 , the expression of all investigated genes were increased significantly compared with that in PBS and pcDNA3.1 groups except MHC II $\beta$, CD4-L2 and CD8 $\alpha$ in pcIL- 8 group, especially IL-1 $\beta$, CXCL10, MHC I $\alpha$ and MHC II $\beta$ gene with more than 4-fold expression (Figure 8). Both pcENO and pcENO+pcIL-8 induced a significantly higher expression of TNF- $\alpha, \mathrm{MHC} \mathrm{I} \alpha$, MHC II $\beta$, CD4-L2 and CD8 $\alpha$ and IFN- $\gamma$ compared with pcIL-8, while the expression of IL-1 $\beta$ and CXCL10 in pcENO group was lower than that of pcIL- 8 group. Moreover, pcENO+pcIL-8 increased the expression of all investigated genes compared with pcENO alone.

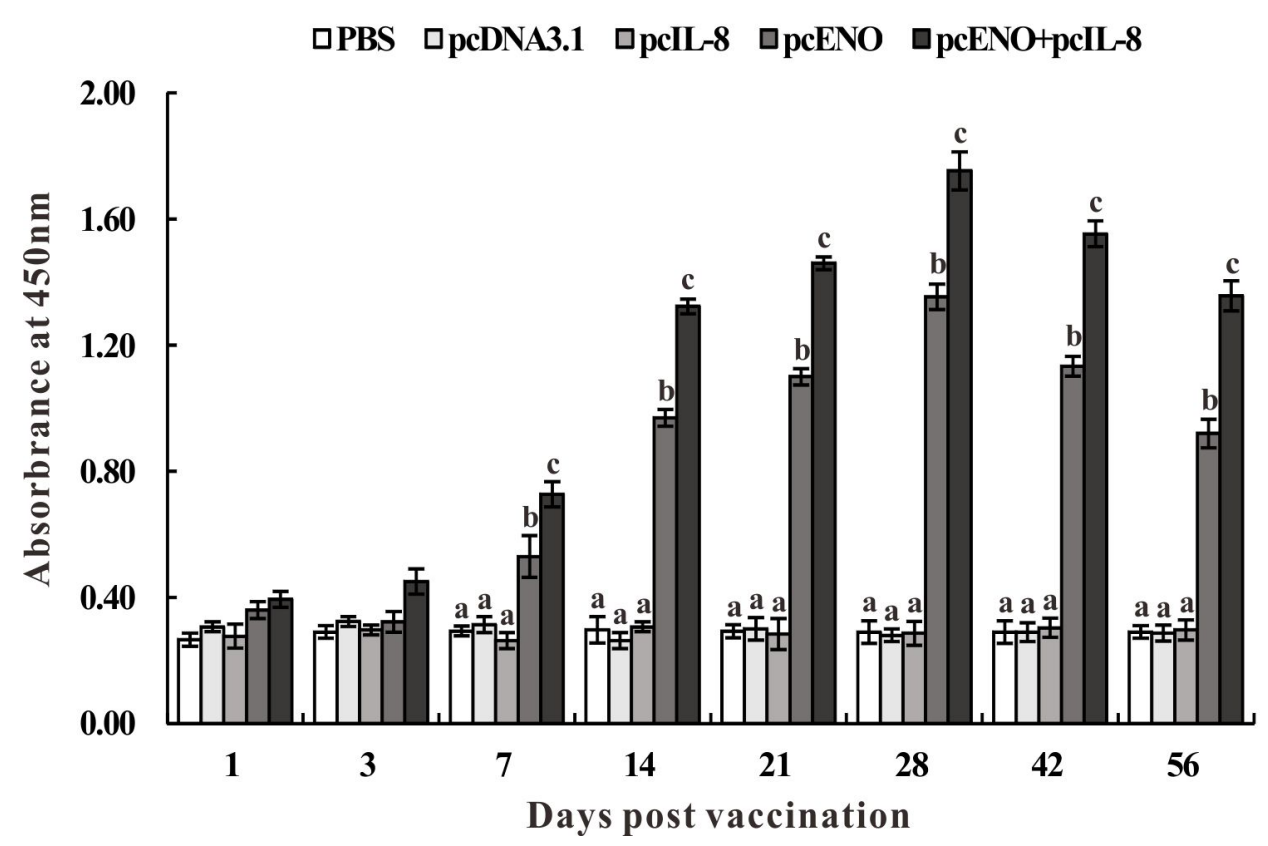

Figure 7: Specific serum antibody detection in vaccinated fish by ELISA. Sera were collected at different days p.v. from the fish vaccinated with PBS (control), pcDNA3.1, pcIL-8, pcENO and pcENO+pcIL-8. Data are presented as means \pm SD $(n=5)$. Different letters mean that the data of different groups in the same time differ significantly $(p<0.05)$.

\section{$\square$ PBS $\square$ pcDNA3.1 $\quad$ apcIL-8 $\square$ pcENO $\square$ pcENO+pcIL-8}

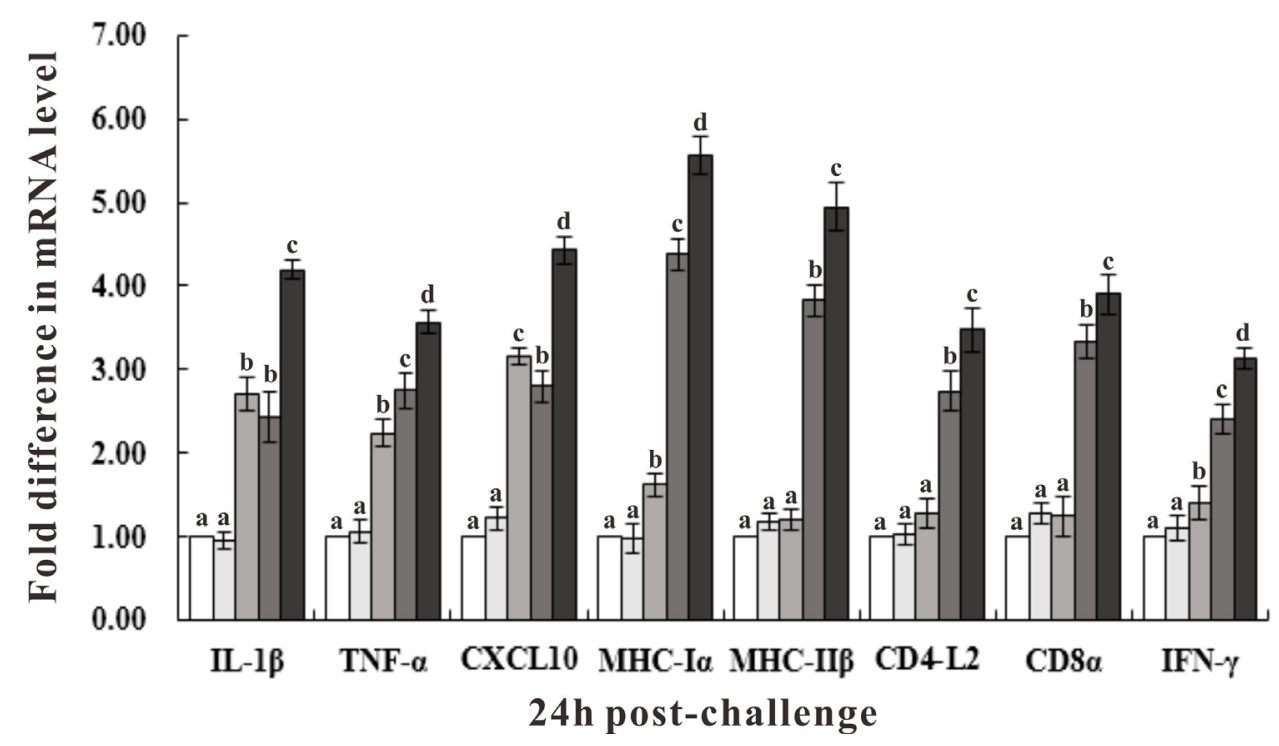

Figure 8: Expression of immune-related genes in vaccinated fish determined by qRT-PCR. Channel catfish were vaccinated with PBS (control), pcDNA3.1, pcIL-8, pcENO and pcENO+pcIL-8. Total RNA was extracted from the head kidney at $24 \mathrm{~h}$ post-challenge of 4-week p.v. and used for qRT-PCR. For each gene, the mRNA level of the PBS-vaccinated fish was set as 1 . Data are presented as means $\pm \mathrm{SD}(n=5)$. Different letters mean that the data of different groups in the same time differ significantly $(p<0.05)$. 


\section{Immune protection against $S$. iniae}

After the challenge experiment with the pathogenic S.iniae DGX07 at 4 weeks p.v, the accumulated survivals of fish vaccinated with PBS, pcDNA3.1, pcIL-8, pcENO and pcENO+pcIL- 8 were $0 \%, 6.67 \%, 20.00 \%, 60.00 \%$ and $80.00 \%$ respectively (Figure $9 \mathrm{~A}$ ), which were equal to the value of corresponding PRS. Moreover, the survivals of group pcENO+pcIL-8 and pcENO were prominently higher $(p<0.05)$ than pcIL-8 group by log-rank test, while the difference of survivals in group pcENO+pcIL-8 and pcENO was not significant (Figure 9B).

To examine the duration of protection, fish were also challenged at 8-week p.v., and the results suggested that the cumulative survivals of fish vaccinated with PBS, pcDNA3.1, pcIL-8, pcENO and pcENO+pcIL-8 were $0 \%, 0 \%, 13.33 \%, 53.33 \%$ and $73.33 \%$ respectively (Figure 9C), which correspond to an RPS of $13.33 \%$ for
pcIL-8 group, $53.33 \%$ for pcENO group and $73.33 \%$ for pcENO+pcIL-8 group respectively. In addition, the results of log-rank test showed that the survival in group pcENO+pcIL-8 and pcENO were remarkably higher $(p<$ 0.05 ) than pcIL-8 group, which is consistent with the results of challenge at 4 weeks p.v, and the difference of survivals in group pcENO+pcIL-8 and pcENO was also significant (Figure 9D). Furthermore, S.iniae DGX07 was the only type of bacterial strain recovered from the liver, and kidney of moribund fish during two challenge tests, suggesting that mortality was caused by S.iniae DGX07 infection.

\section{DISCUSSION}

During the past years, DNA vaccination has proven to be very effective in controlling some devastating viral diseases, but it is not so convincing for other viral

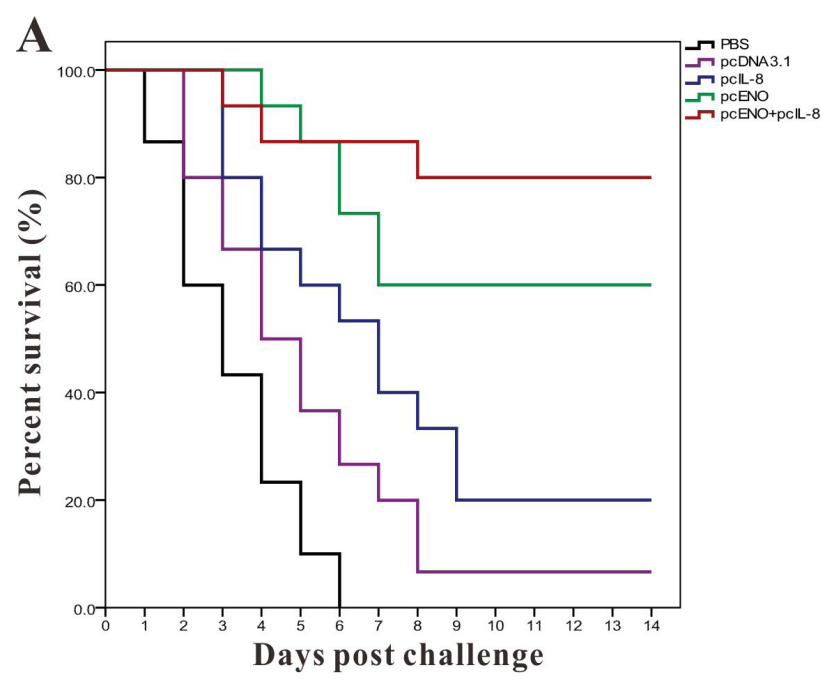

B

\begin{tabular}{ccccc}
\hline \multirow{2}{*}{ Groups } & \multicolumn{4}{c}{ Significance } \\
\cline { 2 - 5 } & PBS & pcDNA3.1 & pcIL-8 & pcENO \\
\hline PBS & \multicolumn{3}{c}{} \\
pcDNA3.1 & $0.002^{*}$ & & & \\
pcIL-8 & $0.000^{*}$ & $0.020^{*}$ & & \\
pcENO & $0.000^{*}$ & $0.000^{*}$ & $0.002^{*}$ \\
pcENO+pcIL-8 & $0.000^{*}$ & $0.000^{*}$ & $0.000^{*}$ & $0.117^{\mathrm{ns}}$ \\
\hline
\end{tabular}

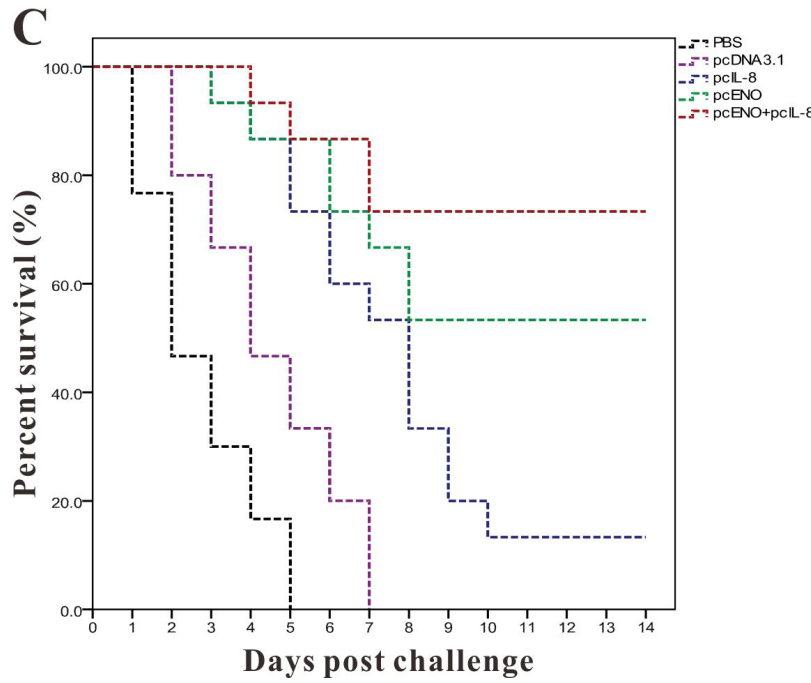

D

\begin{tabular}{lllll}
\hline \multirow{2}{*}{ Groups } & \multicolumn{4}{c}{ Significance } \\
\cline { 2 - 4 } & PBS & pcDNA3.1 & pcIL-8 & pcENO \\
\hline
\end{tabular}

\begin{tabular}{cllll}
\hline PBS & & & & \\
pcDNA3.1 & $0.000^{*}$ & & & \\
pcIL-8 & $0.000^{*}$ & $0.001^{*}$ & & \\
pcENO & $0.000^{*}$ & $0.000^{*}$ & $0.006^{*}$ & \\
pcENO+pcIL-8 & $0.000^{*}$ & $0.000^{*}$ & $0.000^{*}$ & $0.027^{*}$ \\
\hline
\end{tabular}

Figure 9: Percent survivals (Kaplan-Meier) of vaccinated fish $(n=30)$ during the challenges tests of 4 week p.v. A. and 8 week p.v. C.. Differences of survivals among groups were tested using log-rank test shown in B (4 week p.v.) and D (8 week p.v.). The experiments were repeated twice independently with similar results. "**" denotes significant difference $(p<0.05)$, "ns" means not significant. 
infections and bacterial disease. Moreover, to enhance the immune responses and protective efficiency, increasingly focus is now to improve the plasmid vectors and covaccinate with molecular adjuvants such as cytokines in mammals. However, there is very little research related to the capacity of fish cytokines to serve as molecular adjuvants in DNA vaccination against viral and bacterial disease [9]. IL-8, as one of the first CXC chemokines discovered in fish, has been available and sequenced in some fish species, but its immunological role and adjuvant potential which have been proved in mammals $[22,23]$ are rarely reported in fish. In the present study, on the basis of our previous report, we have further explored the capacity of IL-8 as molecular adjuvant by co-injecting with a DNA vaccine against $S$. iniae infection in channel catfish.

Our previous results indicated that IL- 8 hold promise for use as potential immunopotentiator in $\mathrm{rENO}$ subunit vaccine against $S$. iniae infections in channel catfish, but it is insufficient to extend the immune protection for long time [21]. In this study, we first constructed the recombination plasmid pcDNA3.1/IL-8 (pcIL-8), to enhance the transcription and translation efficiency and detect the expression of pcIL-8 in vivo, a Kozak consensus sequence (GCCACCATGG. A purine preferably $A$ in position -3 and a $G$ in position +4 have the strongest effects, modulating translation at least 10fold) and a $6 \times$ histidine tag were insert into the primers for PCR amplication of IL-8. By the identification of gel electrophoresis and sequencing, the target sequence was $360 \mathrm{bp}$ in length encoding a 117 amino acid sequence with a predicted signal peptide (1 24a.a.) and a conserved CXC Chemokine domain (30 88a.a.) containing three consensus motifs named as ELR-like motif, CXC motif and GPH motif respectively, which was different from other fish IL-8 lacking of ELR motifs $[13,14]$ and was belonging to the shorter alternatively spliced transcripts of IL-8 according to Chen et al [17].

Studies in mammals and teleosts have revealed that following intramuscular injection, DNA vaccines are subjected to several fates including localizing extracellularly at the injection site and being uptaken by local cells, degradation or transportation to other tissues $[24,25]$. To determine whether the plasmid DNA was present and transcribed in vivo, DNA and RNA was extracted from the muscle tissues around the injection site for PCR and RT-PCR detection respectively. To detect the expression of pcIL-8 at translation levels in muscle of vaccinated fish, immunohistochemistry analysis was performed. The results showed that target genes (ENO gene and exogenous IL-8 gene) were detected in muscle tissue, suggesting that some plasmid DNA had escaped the fate of degradation and been uptaken by local cells, following by being expressed at transcription levels. Consistent with above results, the positive signals of exogenous IL-8 were detected in muscle of pcIL- 8 and pcENO+pcIL-8 group by immunohistochemistry, which suggested exogenous IL-8 were expressed successfully in these groups, while no positive signals in muscle of other groups. These observations are similar to those reported previously in other fish species such as Japanese flounder $[25,26]$, rainbow trout [27], Atlantic cod [28] and Atlantic salmon [29].

Innate immunity as a fundamental defence mechanism plays an instructive role in the immune system and homeostasis in fish $[30,31]$. Lysozyme activity and alternative complement activity $\left(\mathrm{ACH}_{50}\right)$ are two important parameters to evaluate the host innate immune response and both are involved in activating the complement system and defending against bacterial pathogens [31-34]. The results of the present study showed that both pcIL-8 and pcENO increased the level of serum lysozyme activity and $\mathrm{ACH}_{50}$ activity compared with PBS and pcDNA3.1, which induced the innate immune responses and activated the protective mechanisms, resulting in higher level of lysozyme activity and ACH50 activity appearing in fish vaccinated with pcENO+pcIL-8. It is speculated that co-injection of pcIL- 8 could stimulate the NF-kB/IL-8 signaling pathway in response to inflammation, and the released NF-kB translocates into the nucleus where it activates the transcription or function of downstream immune-related molecules like lysozyme and complement components $[35,36]$, which together with cytokines and chemokines are the important humoral components for the innate immune system [31]. The difference of these two indexes was that the highest lysozyme activity located in pcENO+pcIL-8 group at 21 days p.v. while the highest ACH50 activity was also detected in pcENO+pcIL-8 group but at 14 days p.v.

Specific antibody induced by subunit or DNA vaccines had been proven to be the basic mechanism of immunoprotection against $S$. iniae infection and been observed in various fish models [25, 26, 37-39]. In this study, pcENO and pcENO+pcIL-8 significantly stimulated the production of anti-rENO specific antibody which were detected at 7 days p.v. and maintained for 56 days p.v. compared with PBS, pcDNA3.1 and pcIL8 group. Moreover, the antibody level of fish vaccinated with pcENO+pcIL-8 was remarkably higher than that of pcENO from 7 to 56 days p.v. with the peak of antibody titers both appearing at 28 days p.v., which indicated that pcIL- 8 functioned for increasing the antibody level induced by pcENO vaccination. However, the time of antibody production and the peak in our study was different or even brought forward comparing with other vaccines against the same pathogen [38-41], chiefly because of the different vaccines and fish species, as well as different immune schedules.

Despite specific antibody is important for the immune response against $S$. iniae infection, it is not sufficient for complete protection which involves both humoral and cellular immunity [42]. Many immunerelated molecules or genes related to pro-inflammatory 
response, humoral immunity and cellular immunity are also contribute to the immune protection. Therefore, transcriptional analysis of immune-related genes including IL-1 $\beta$, TNF- $\alpha$, CXCL10, MHC I $\alpha$, MHC II $\beta$, CD4-L2, CD $8 \alpha$ and IFN- $\gamma$ were conducted by qRT-PCR in our study. The results showed that compared with PBS and pcDNA3.1, pcENO and pcIL-8 increased the expression of all investigated genes especially MHC I $\alpha$, MHC II $\beta$, CD $8 \alpha$ in pcENO group and IL-1 $\beta$, TNF- $\alpha$, CXCL10 in pcIL-8 group, which was consistent with the observation that co-administration of pIL8+ with pMCV1.4-G activated the cytokine response and increased the expression of two pro-inflammatory cytokines (IL-1 $\beta$ and TNF- $\alpha$ ) in head kidney of rainbow trout [43]. Moreove, basing on this upregulation, pcENO+pcIL-8 notably induced the expression of all investigated genes compared with pcENO alone (Figure 8). These results shown above suggested that not only pcENO could induce a systemic immune response, but also pcIL- 8 could activate inflammatory response and strengthen the immune response in channel catfish against $S$. iniae infection.

RPS as a measure to evaluate vaccine efficacy against pathogen infection, is one of the most visual indices to assess the immuneprotection effect in challenge test [42]. In the present study, pcENO induced a moderate immune protection (with RPS of 60\%) for channel catfish against S.iniae infection in the challenge experiment of 4 weeks p.v., which was slightly higher than the RPS (50\%) of rENO subunit vaccine in our previous study [21] and lower than the RPS (100\%) of rENO in zebrafish against same pathogen [39], mainly due to different vaccine types and vaccine delivery route, as well as different fish species and infection dosages. As expected, co-vaccination with pcIL-8 enhanced the protection efficacy of pcENO, resulting in the RPS of $80 \%$ in pcENO+pcIL-8 group, which was higher but not significant comparing with the RPS $(60 \%)$ of pcENO alone by log-rank test at the same time. However, pcENO and pcENO+pcIL-8 could still provide the RPS of $53.33 \%$ and $73.33 \%$ respectively at the challenge of at 8 weeks p.v. , which were comparable with other DNA vaccines such as pSia10 with RPS of $73.9 \%$ in turbot [38] and pSagF with RPS of 78\% in Japanese flounder [25] against S.iniae at 4 weeks p.v. Moreover, the RPS in group pcENO+pcIL- 8 and pcENO showed significant difference $(p<0.05)$.

Taken together, these results indicated that pcIL8 served as molecular adjuvant co-injection with DNA vaccine not only improve the immune protection but also maintain long period of immunity for channel catfish against S.iniae infection. Our study signified that IL-8 hold promise to serve as a potential adjuvant in DNA vaccines against bacterial infections in fish.

\section{MATERIALS AND METHODS}

\section{Ethics statement}

All animal experiments were reviewed and approved by the Committee of the Ethics on Animal Care and Experiments at Sichuan Agricultural University. All experimental procedures were perfomed in accordance with the Guidelines for Experimental Animals maintained by Chinese Ministry of Science and Technology.

\section{Bacterial strains, plasmids, reagents and growth conditions}

S.iniae DGX07 is a pathogenic isolate from diseased channel catfish in China and stored at our laboratory, it was cultured in Brain-Heart Infusion (BHI) medium at $37^{\circ} \mathrm{C}$. E. coli $\mathrm{DH} 5 \alpha$ were used as the host strains for cloning which were routinely grown in Luria-Bertani (LB) medium containing $100 \mu \mathrm{g} / \mathrm{ml}$ of ampicillin at $37^{\circ} \mathrm{C}$. The plasmids pMD19-T simple (Takara, Japan) and pcDNA3.1 $(+)$ (Invitrogen, USA) were used for T-A cloning and eukaryotic expression, respectively. The recombinant DH5 $\alpha$ containing pcDNA3.1-ENO (pcENO) plasmid and recombinant protein $\mathrm{rENO}$ were constructed and stored at our laboratory [44].

\section{Primers design, amplification and cloning of IL-8}

In order to enhance the transcription and translation efficiency of IL-8 gene in eukaryotic expression, a Kozak consensus sequence (GCCACCATGG) [45] was added at the 5 ' end of the front primers. Besides, a $6 \times$ GTG sequence (reverse complement of $6 \times$ histidine tag) was also insert at the 5 ' end of the reverse primers for the detection of IL-8 expression in vivo. The primers were designed based on the channel catfish IL-8 sequence published in GenBank (KP701473) and named as IL-8F (5-CCC AAGCTTGCCACCATGGAGGCTGCAACTCTCACA -3 , underlined shown as Hind III site, bold shown as Kozak consensus sequence) and IL-8R1 (5-GGAATTCTCA GTGGTGGTGGTTTTGCTGTTTGATCTTCT-3, underlined shown as EcoR I site, bold shown as reverse complement of $6 \times$ histidine tag). The primers IL-8F and IL-8R2 (5-GTGGTGGTGGTGGTGGTGGT-3) were used for the detection of target plasmid DNA in vivo.

Total RNA was extracted from head kidney of healthy channel catfish with RNAiso Plus Kit (TaKaRa, Dalian, China) and was reverse-transcribed into firststrand cDNA using PrimeScrip $\mathrm{t}^{\mathrm{TM}}$ RT reagent Kit with gDNA Eraser (Perfect Real Time) (TaKaRa) according to the manufacturer's instructions. The target gene IL-8 was amplified by PCR with the cDNA template and primers 
IL-8F/IL-8R under the following conditions: 1 cycle of $95^{\circ} \mathrm{C}$ for $5 \mathrm{~min}, 30$ cycles of $95^{\circ} \mathrm{C}$ for $1 \mathrm{~min}, 57.6^{\circ} \mathrm{C}$ for $30 \mathrm{~s}$, and $72^{\circ} \mathrm{C}$ for $30 \mathrm{~s}$, followed by a final extension of $72^{\circ} \mathrm{C}$ for $10 \mathrm{~min}$. Then the PCR products were identified on $1 \%$ agarose gels, purified using the Agarose Gel DNA Extraction Kit (TaKaRa) and cloned into the pMD19-T vector, followed by transformation into $E$. coli $\mathrm{DH} 5 \alpha$. The positive recombinant clone was selected using an Amp/ IPTG/X-Gal agar plate. The plasmid was extracted from the positive recombinant clone using SanPrep Column Plasmid Mini-Preps Kit (Sangon Biotech, Shanghai, China), identified by PCR under the aforementioned conditions, digested with restriction enzymes Hind III and EcoR I, and fractionated on 1\% agarose gels. DNA sequencing was conducted by Sangon Biotech and the correct recombinant plasmid was named as T-IL-8.

\section{Plasmid construction and preparation}

The plasmid T-IL-8 was digested with Hind III and EcoR I and the IL-8-containing fragment was retrieved and inserted into Hind III /EcoR I-digested pcDNA3.1 (+) vector with T4 Ligase (TAKARA), resulting in recombinant plasmid pcDNA3.1/IL-8 (pcIL-8). The plasmid pcIL-8 was also transformed into E. coli $\mathrm{DH} 5 \alpha$, identified and sequenced as above described. Endotoxinfree plasmid DNA was extracted using GoldHi EndoFree Plasmid Maxi Kit (Kangwei, Beijing, China) according to the manufacturer's instructions. The plasmid DNA (pcDNA3.1, pcIL-8 and pcENO) concentration and purity were determined by Nanodrop 2000 (Thermo Scientific, Wilmington, DE, USA) with measuring absorbance at $\mathrm{A}_{260}$ and $\mathrm{A}_{260} / \mathrm{A}_{280}$.

\section{Preparation of fish and vaccines}

Channel catfish $(50.0 \mathrm{~g} \pm 5.0 \mathrm{~g})$ were purchased from a fish farm in Chengdu (Sichuan, China) and acclimatized in the laboratory for 2 weeks before experimental manipulation. The fish were fed a commercial diet daily, and the water was partly replaced every day, maintaining a temperature of $28^{\circ} \mathrm{C} \pm 1^{\circ} \mathrm{C}$. Before experiments, fish were randomly sampled from blood, liver, kidney, and spleen, the examination of bacterial recovery indicated no bacteria could be detected and agglutination test showed no reaction between the serum and $S$. iniae DGX07. Fish were anaesthetized with tricaine methanesulfonate (MS222) (Sigma, Beijing, China) prior to injections and blood collection. The plasmid pcDNA3.1, pcIL-8 and pcENO were diluted in PBS to $250 \mu \mathrm{g} / \mathrm{ml}$, to obtain pcENO+pcIL-8, the plasmid pcENO was mixed with an equal volumes of pcIL-8.

\section{Vaccination and bacterial challenge}

Five hundred channel catfish were divided randomly into five groups (100 fish /group) and injected intramuscularly (i.m.) with $100 \mu \mathrm{l}$ of PBS (control), pcDNA3.1, pcIL-8, pcENO and pcENO+pcIL-8. At 4 weeks and 8 weeks post vaccination (p.v.). 30 fish from each group were randomly selected and challenged by intraperitoneal injection (i.p.) with $0.2 \mathrm{ml}$ of the $S$. iniae strain DGX07 that resuspended in PBS to $6 \times 10^{7} \mathrm{CFU} / \mathrm{ml}$ [46]. Mortality was monitored over a period of 14 days after the challenge, and dying fish were randomly selected for the examination of bacterial recovery from liver, kidney, and spleen. Relative percent of survival (RPS) was calculated according to the following formula: RPS = [1- (\% mortality of vaccinated fish $/ \%$ mortality of control fish)] $\times 100$ [47]. Serum samples of five fish in each group were collected for assessment of immune related indexes at 1, 3, 7, 14, 21, 28, 42 and 56 days p.v., and head kidney of five fish were taken for qRT-PCR at $24 \mathrm{~h}$ post-challenge of 4 week p.v. All vaccination trials were repeated once.

\section{PCR detection of plasmid DNA in vaccinated fish}

Muscle tissues around the injection site of five fish in each group were taken at 7, 14, 28 and 56 days p.v. DNA was extracted from the tissues with MiniBEST Universal Genomic DNA Extraction Kit (TAKARA). PCR was conducted using specific primers (IL-8F/IL-8R2) to plasmid DNA.

\section{Reverse transcriptase-PCR (RT-PCR) analysis of expression of DNA vaccines}

Total RNA was extracted from muscle tissues around the injection site of five fish in each vaccinated group at 14 days p.v. using RNAiso Plus Kit (TaKaRa) and was reverse-transcribed into first-strand cDNA as described above. About 360 bp product of exogenous IL-8 gene segment was amplified with the specific primers IL$8 \mathrm{~F} / \mathrm{IL}-8 \mathrm{R} 2$ and from the cDNA by RT-PCR using $\beta$-actin (ACTB) as an internal control, as well as the amplification of the ENO gene ( $\sim 1320 \mathrm{bp})$ with the specific primers as described previously [44]. The RT-PCR products were electrophoresed on a $1 \%$ agarose gel.

\section{Immunohistochemical analysis of exogenous IL-8 expression in vivo}

Immunohistochemistry was performed according to the method of Seo et al. [48] with some modification. Briefly, muscle tissues were taken from five vaccinated fish in each group at 14, 28 and 56 days p.v. The tissue samples were fixed with $4 \%$ paraformaldehyde for $48 \mathrm{~h}$, 
followed by dehydrating in ethanol and embedding in paraffin. The sections $(5 \mu \mathrm{m})$ were cut with a Leica RM2135 microtome (Leica Microsystems, Germany), and incubating with rabbit anti-6-histidine monoclonal antibody (Sangon Biotech, Shanghai, China) and then with goat-anti-rabbit IgG $(\mathrm{H}+\mathrm{L})$-HRP (Sigma, St. Louis, MO, USA). After staining with DAB (Sigma) for 5 to $15 \mathrm{~min}$, the sections were observed by light microscopy (Nikon, Tokyo, Japan).

\section{Detection of innate immune parameters}

Serum lysozyme activity was measured by the turbidimetric method as described previously [21]. Briefly, $150 \mu \mathrm{L}$ Micrococcus lysodeikticus with a concentration of $0.2 \mathrm{mg} / \mathrm{mL}$ (in $0.04 \mathrm{M}$ PBS, $\mathrm{pH}=6.2$ ) was added to $15 \mu \mathrm{L}$ sera in a 96-well U-bottom microtiter plate, quickly mixed by vortex, and the OD520 (UV absorption value at 520 $\mathrm{nm})\left(\mathrm{A}_{0}\right)$ was assayed at 0.5 and $4.5 \mathrm{~min}$ after reaction, respectively. Each test was conducted in triplicate. One activity unit of lysozyme (U) was defined as the amount of serum lysozyme that caused a decrease in absorbancy of 0.001 per min at $520 \mathrm{~nm}$.

Alternative complement activity (ACH50) was determined based on haemolysis of rabbit red blood cells (RaRBC) as described by Sunyer and Tort [49]. The volume of serum yielding 50\% haemolysis (ACH50) was determined and used to calculate the complement activity of the samples (value of ACH50 is in units per ml)

\section{Enzyme-linked immunosorbent assay (ELISA)}

Specific anti-rENO antibody were determined by ELISA as described previously [21]. Briefly, the rENO was diluted to a concentration of $50 \mu \mathrm{g} / \mathrm{mL}$ in a carbonate buffer ( $\mathrm{pH}=9.6$ ). Each well of the 96-well plate was covered with $100 \mu \mathrm{L}$ diluted rENO overnight at $4^{\circ} \mathrm{C}$ followed by washing with PBST (0.1\% Tween-20 in PBS) and blocking with $3 \% \mathrm{BSA}$ in PBST for $2 \mathrm{~h}$ at $37^{\circ} \mathrm{C}$. Serial 2 -fold dilutions of sera were added to the wells in triplicate and subsequently incubated for $2 \mathrm{~h}$ at $37^{\circ} \mathrm{C}$. Rabbit antichannel catfish IgM antibody (prepared in our laboratory) (1:2000) and goat-anti-rabbit IgG $(\mathrm{H}+\mathrm{L})$-HRP (1:2000) were used as the secondary and tertiary antibodies, respectively. Color development was performed with the TMB kit (Tiangen, Beijing, China). The plates were read at $450 \mathrm{~nm}$ with a microplate reader (Bio-Rad, Hercules, USA).

\section{qRT-PCR analysis of the expression of immune- related genes}

Head kidney samples were taken from the fish (five in each group) at $24 \mathrm{~h}$ post-challenge of 4 weeks p.v. Total RNA extraction and cDNA synthesis were carried out as described above. qRT-PCR was performed with the SYBR ${ }^{\circledR}$ Premix Ex Taq ${ }^{\mathrm{TM}}$ II (Tli RNaseH Plus) (TaKaRa) in an ABI StepOnePlus ${ }^{\text {TM }}$ System (Applied Biosystems, USA) as described previously [50]. Each assay was performed in triplicate with $\beta$-actin (ACTB) as an internal control. The primers used to amplify the immune-related genes have been reported and shown in our previous research [21]. All data are given in terms of relative mRNA.

\section{Statistical analysis}

All statistical analyses were performed with SPSS 19.0 software (SPSS Inc., USA). Mortality data from the challenge experiments were analyzed by the Kaplan-Meier methods and differences among groups were tested using log-rank test. The difference significance of other data were determined using a one-way analysis of variance (ANOVA). In all cases, the significance level was defined as $p<0.05$ and the results were presented as means \pm SD (standard deviation).

\section{ACKNOWLEDGMENTS}

This work was supported by the Program for Changjiang Scholars and Innovative Research Team in University (No. IRT0848), Sichuan Technology Support Planning (No.2014NZ0003) and (No. 2014JY0143).

\section{CONFLICTS OF INTEREST}

The authors declare no conflict of interest.

\section{REFERENCES}

1. Evensen $\varnothing$, Leong JC. DNA vaccines against viral diseases of farmed fish. Fish Shellfish Immunol. 2013; 35:17511758.

2. Leong JA, Romoren K, Evensen O. DNA vaccines for viral diseases of farmed fish and shellfish. Aquaculture biotechnology. Wiley. 2012. 153-173

3. Rajeshkumar S, Venkatesan C, Sarathi M, Sarathbabu V, Thomas J, Basha KA, Hameed AS. Oral delivery of DNA construct using chitosan nanoparticles to protect the shrimp from white spot syndrome virus (WSSV). Fish Shellfish Immunol. 2009; 26:429-437.

4. Kumar SR, Ahamed VI, Sarathi M, Basha AN, Hameed AS. Immunological responses of Penaeus monodon to DNA vaccine and its efficacy to protect shrimp against white spot syndrome virus (WSSV). Fish Shellfish Immunol. 2008; 24:467-478.

5. Emmenegger EJ, Kurath G. DNA vaccine protects ornamental koi (Cyprinus carpio koi) against North 
American spring viremia of carp virus. Vaccine. 2008; 26:6415-6421.

6. Takano T, Iwahori A, Hirono I, Aoki T. Development of a DNA vaccine against hirame rhabdovirus and analysis of the expression of immune-related genes after vaccination. Fish Shellfish Immunol. 2004; 17:367-374.

7. Kanellos T, Sylvester ID, D Mello F, Howard CR, Mackie A, Dixon PF, Chang K, Ramstad A, Midtlyng PJ, Russell PH. DNA vaccination can protect Cyprinus Carpio against spring viraemia of carp virus. Vaccine. 2006; 24:49274933.

8. Kutzler MA, Weiner DB. DNA vaccines: ready for prime time? Nat Rev Genet. 2008; 9:776-788.

9. Lorenzen N, LaPatra SE. DNA vaccines for aquacultured fish. Rev. Sci. Tech. Off. Int. Epiz. 2005; 24: 201-213.

10. Kurath G. Overview of recent DNA vaccine development for fish. Dev. Biol. (Basel). 2004; 121:201-213.

11. Secombes CJ, Zou J, Bird S. Fish cytokines: discovery, activities and potential applications. Fish Defenses. 2009; 1:1-36.

12. Laing KJ, Secombes CJ. Chemokines. Dev Comp Immunol. 2004; 28:443-460.

13. Najakshin AM, Mechetina LV, Alabyev BY, Taranin AV. Identification of an IL-8 homolog in lamprey( Lampetra fluviatilis): early evolutionary divergence of chemokines. Eur J Immunol. 1999; 29:375-382.

14. Lee E, Park H, Kim Y, Chung J, Choi T. Cloning and sequence analysis of the interleukin-8 gene from flounder (Paralichthys olivaceous). Gene. 2001; 274:237-243.

15. Huising MO, Stolte E, Flik G, Savelkoul HF, Verburg-van Kemenade BL. CXC chemokines and leukocyte chemotaxis in common carp (Cyprinus carpio L.). Dev Comp Immunol. 2003; 27:875-888.

16. Corripio-Miyar Y, Bird S, Tsamopoulos K, Secombes CJ. Cloning and expression analysis of two pro-inflammatory cytokines, IL-1 $\beta$ and IL-8, in haddock (Melanogrammus aeglefinus). Mol Immunol. 2007; 44:1361-1373.

17. Chen L, He C, Baoprasertkul P, Xu P, Li P, Serapion J, Waldbieser G, Wolters W, Liu Z. Analysis of a catfish gene resembling interleukin-8: cDNA cloning, gene structure, and expression after infection with Edwardsiella ictaluri. Dev Comp Immunol. 2005; 29:135-142.

18. Shoemaker CA, Klesius PH, Evans JJ. Prevalence of Streptococcus iniae in tilapia, hybrid striped bass, and channel catfish on commercial fish farms in the United States. Am J Vet Res. 2001; 62:174-177.

19. Evans JJ, Klesius PH, Pasnik DJ, Shoemaker CA. Influence of natural Trichodina sp. parasitism on experimental Streptococcus iniae or Streptococcus agalactiae infection and survival of young channel catfish Ictalurus punctatus (Rafinesque). Aquac Res. 2007; 38:664-667.

20. Chen D, Wang K, Geng Y, Wang J, Huang L, Li J. Streptococcus iniae isolated from channel catfish (Ictalurus punctatus) in China. Isr J Aquacult-Bamid. 2011; 63: 593-
599

21. Wang E, Wang J, Long B, Wang K, He Y, Yang Q, Chen D, Geng Y, Huang X, Ouyang P. Molecular cloning, expression and the adjuvant effects of interleukin- 8 of channel catfish (Ictalurus Punctatus) against Streptococcus iniae. Sci Rep-Uk. 2016; 6.

22. Kim JJ, Yang J, Dentchev T, Dang K, Weiner DB. Chemokine gene adjuvants can modulate immune responses induced by DNA vaccines. J Interferon Cytokine Res. 2000; 20:487-498.

23. Min W, Lillehoj HS, Burnside J, Weining KC, Staeheli P, Zhu JJ. Adjuvant effects of IL-1 $\beta$, IL-2, IL-8, IL-15, IFN- $\alpha$, IFN- $\gamma$, TGF- $\beta 4$ and lymphotactin on DNA vaccination against Eimeria acervulina. Vaccine. 2001; 20:267-274.

24. Tonheim TC, Bøgwald J, Dalmo RA. What happens to the DNA vaccine in fish? A review of current knowledge. Fish Shellfish Immunol. 2008; 25:1-18.

25. Sun Y, Hu Y, Liu C, Sun L. Construction and comparative study of monovalent and multivalent DNA vaccines against Streptococcus iniae. Fish Shellfish Immunol. 2012; 33:1303-1310.

26. Sun Y, Zhang M, Liu C, Qiu R, Sun L. A divalent DNA vaccine based on Sia10 and $\mathrm{OmpU}$ induces cross protection against Streptococcus iniae and Vibrio anguillarum in Japanese flounder. Fish Shellfish Immunol. 2012; 32:12161222.

27. Boudinot $\mathrm{P}$, Blanco $\mathrm{M}$, de Kinkelin $\mathrm{P}$, Benmansour A. Combined DNA immunization with the glycoprotein gene of viral hemorrhagic septicemia virus and infectious hematopoietic necrosis virus induces double-specific protective immunity and nonspecific response in rainbow trout. Virology. 1998; 249:297-306.

28. Seternes T, Tonheim TC, Løvoll M, Bøgwald J, Dalmo RA. Specific endocytosis and degradation of naked DNA in the endocardial cells of cod (Gadus morhua L.). J Exp Biol. 2007; 210:2091-2103.

29. Tonheim TC, Dalmo RA, Bøgwald J, Seternes T. Specific uptake of plasmid DNA without reporter gene expression in Atlantic salmon (Salmo salar L.) kidney after intramuscular administration. Fish Shellfish Immunol. 2008; 24:90-101.

30. Zhu L, Nie L, Zhu G, Xiang L, Shao J. Advances in research of fish immune-relevant genes: a comparative overview of innate and adaptive immunity in teleosts. Dev Comp Immunol. 2013; 39:39-62.

31. Magnadóttir B. Innate immunity of fish (overview). Fish Shellfish Immunol. 2006; 20:137-151.

32. Swain P, Dash S, Sahoo PK, Routray P, Sahoo SK, Gupta SD, Meher PK, Sarangi N. Non-specific immune parameters of brood Indian major carp Labeo rohita and their seasonal variations. Fish Shellfish Immunol. 2007; 22:38-43.

33. Wang Z, Zhang S, Tong Z, Li L, Wang G. Maternal transfer and protective role of the alternative complement components in zebrafish Danio rerio. Plos One. 2009; 4:e4498. 
34. Wang Z, Zhang S. The role of lysozyme and complement in the antibacterial activity of zebrafish (Danio rerio) egg cytosol. Fish Shellfish Immunol. 2010; 29:773-777.

35. Gilmore TD. Introduction to NF- $\mathrm{BB}$ : players, pathways, perspectives. Oncogene. 2006:6680-6684.

36. Végran F, Boidot R, Michiels C, Sonveaux P, Feron O. Lactate influx through the endothelial cell monocarboxylate transporter MCT1 supports an NF-kB/IL-8 pathway that drives tumor angiogenesis. Cancer Res. 2011; 71:25502560 .

37. Agnew W, Barnes AC. Streptococcus iniae: an aquatic pathogen of global veterinary significance and a challenging candidate for reliable vaccination. Vet Microbiol. 2007; 122:1-15.

38. Sun Y, Hu Y, Liu C, Sun L. Construction and analysis of an experimental Streptococcus iniae DNA vaccine. Vaccine. 2010; 28:3905-3912.

39. Membrebe JD, Yoon N, Hong M, Lee J, Lee H, Park K, Seo S, Yoon I, Yoo S, Kim Y. Protective efficacy of Streptococcus iniae derived enolase against Streptococcal infection in a zebrafish model. Vet Immunol Immunop. 2016; 170, 25-29

40. Cheng S, Hu Y, Jiao X, Sun L. Identification and immunoprotective analysis of a Streptococcus iniae subunit vaccine candidate. Vaccine. 2010; 28:2636-2641.

41. Zou L, Wang J, Huang B, Xie M, Li A. MtsB, a hydrophobic membrane protein of Streptococcus iniae, is an effective subunit vaccine candidate. Vaccine. 2011; 29:391394.

42. Costa AA, Leef MJ, Bridle AR, Carson J, Nowak BF. Effect of vaccination against yersiniosis on the relative percent survival, bactericidal and lysozyme response of Atlantic salmon, Salmo salar. Aquaculture. 2011; 315:201-206.

43. Jimenez N, Coll J, Salguero FJ, Tafalla C. Co-injection of interleukin 8 with the glycoprotein gene from viral haemorrhagic septicemia virus (VHSV) modulates the cytokine response in rainbow trout (Oncorhynchus mykiss). Vaccine. 2006; 24:5615-5626.
44. Wang J, Wang K, Chen D, Geng Y, Huang X, He Y, Ji L, Liu T, Wang E, Yang Q. Cloning and characterization of surface-localized $\alpha$-enolase of Streptococcus iniae, an effective protective antigen in mice. Int J Mol Sci. 2015; 16:14490-14510.

45. Kozak M, Evans M, Gardner PD, Flores I, Mariano TM, Pestka S, Phelps A, Wohlrab H, Zushi M, Gomi K. Structural features in eukaryotic mRNAs that mod. Biol Chem. 1991; 266:19867-19870.

46. Jiang J, Zheng Z, Wang K, Wang J, He Y, Wang E, Chen D, Ouyang P, Geng Y, Huang X. Adjuvant Immune Enhancement of Subunit Vaccine Encoding pSCPI of Streptococcus iniae in Channel Catfish (Ictalurus punctatus). Int J Mol Sci. 2015; 16:28001-28013.

47. Amend DF. Potency testing of fish vaccines. Fish biologics: serodiagnostics and vaccines. 1981; 49: 447-454

48. Seo JY, Kim K, Kim S, Oh M, Nam S, Kim Y, Choi T. Protection of flounder against hirame rhabdovirus (HIRRV) with a DNA vaccine containing the glycoprotein gene. Vaccine. 2006; 24:1009-1015.

49. Sunyer JO, Tort L. Natural hemolytic and bactericidal activities of sea bream Sparus aurata serum are effected by the alternative complement pathway. Vet Immunol Immunop. 1995; 45:333-345.

50. Wang E, Wang K, Chen D, Wang J, He Y, Long B, Yang L, Yang Q, Geng Y, Huang X. Evaluation and selection of appropriate reference genes for real-time quantitative pcr analysis of gene expression in nile tilapia (Oreochromis niloticus) during vaccination and infection. Int J Mol Sci. 2015; 16:9998-10015. 